\title{
Resfriamento de um cilindro de aço: estudo experimental da convecção e radiação do calor
}

\author{
Cooling of a steel cylinder: experimental study of convection and heat radiation
}

\author{
R. L. Garcia ${ }^{1}$, R. A. Amaral², J. Zabadal'², C. C. Pibernat ${ }^{1}$, F. Juchem ${ }^{1}$, A. Schmitz \\ ${ }^{1}$ Engenharia de Biotecnologia e Bioprocessos, Universidade Estadual do Rio Grande do Sul, Novo Hamburgo, RS, Brasil. \\ ${ }^{2}$ Departamento Interdisciplinar, Universidade Federal do Rio Grande do Sul, Tramandaí, RS, Brasil.
}

Recebido em 31 de Janeiro de 2017. Revisado em 16 de Março de 2017. Aceito em 4 de Abril de 2017

\begin{abstract}
Nesse trabalho é descrito um experimento quantitativo destinado a avaliar a transferência de calor durante o resfriamento de um cilindro de aço e comparar os resultados experimentais com valores previstos em modelos de convecção e radiação do calor. Além de executar os ensaios, o aluno é requisitado a confeccionar uma planilha eletrônica que detalha os cálculos realizados e avalia a consistência dos resultados. O experimento foi concebido para ser facilmente implementado em aulas práticas de cursos de graduação; e, nesse intuito, buscou-se utilizar materiais e equipamentos que viabilizem a realização de ensaios simples e de fácil execução. Os resultados obtidos são satisfatórios e propiciam uma análise quantitativa sobre os mecanismos envolvidos na transferência de calor num sistema físico real.
\end{abstract}

Palavras-chave: Transferência de calor, resfriamento de um cilindro de aço, convecção, radiação.

This work describes a quantitative experiment to evaluate the heat transfer during the cooling of a steel cylinder and compare the experimental results with predicted values in convection and heat radiation models. In addition to performing the tests, the student is required to prepare a spreadsheet that details the calculations performed and evaluates the consistency of the results. The experiment was designed to be easily implemented in experimental classes of undergraduate courses; and, for this, we tried to use materials and equipment that make feasible the realization of simple and easy execution tests. The results obtained are satisfactory and provide a quantitative analysis on heat transfer mechanisms involved in a real physical system.

Keywords: Heat transfer, cooling of a steel cylinder, convection, radiation.

\section{Introdução}

As disciplinas de Fenômenos de Transporte são fundamentais na formação de um engenheiro; pois, sem a correta interpretação e equacionamento dos processos físicos nelas abordado, o aluno não estará apto ao aprendizado das Operações Unitárias, do Cálculo de Reatores, de Trocadores de Calor e outras disciplinas aplicadas a processos industriais $[1,2]$.

A extensão do conteúdo programático, que inclui a análise de uma série de situações específicas envolvendo diversas equações, muitas das quais de natureza empírica, pode constituir um obstáculo ao aprendizado dessas disciplinas. Um dos principais objetivos das disciplinas aplicadas de um curso de Engenharia, a exemplo de Fenômenos de Transporte, é dotar o aluno da capacidade de aplicar o conhecimento adquirido a uma ampla variedade de problemas práticos. Nesse contexto, o aluno deverá ser capaz de efetuar um tipo de análise, característica de um engenheiro, na qual se busca identificar as informações mais

*Endereço de correspondência: rafael.amaral@ufrgs.br relevantes referentes a um projeto ou ao desempenho de um certo sistema ou processo [3].

Uma das formas de motivar o aluno na busca desse objetivo, evitando a mera abordagem teórico-expositiva dessas equações, é a realização de aulas práticas que possibilitem a visualização do fenômeno físico objeto de estudo e a quantificação numérica dos seus efeitos [4]. O uso de equipamentos simples e de baixo custo aliado à realização de ensaios de fácil execução constitui uma alternativa interessante para a implementação desse tipo de aula em instituições de ensino que enfrentam dificuldades financeiras ou entraves burocráticos para a aquisição de equipamentos de laboratório [5, 6].

Nesse artigo é descrito um experimento didático, destinado ao estudo da transferência de calor, que apresenta as características citadas no parágrafo anterior. O resfriamento de um cilindro de aço, exposto ao ar ambiente e cuja temperatura é monitorada, é analisado com base num balanço térmico. Os resultados obtidos são comparados com modelos teóricos que estimam o calor transferido por convecção e radiação. 


\section{Fundamentação teórica}

A transferência de calor na interface que delimita um corpo sólido e um fluido circundante é um fenômeno cuja quantificação é fundamental no projeto de caldeiras, máquinas térmicas, trocadores de calor e diversos outros equipamentos industriais. Modelos baseados nas leis de Fourier, de resfriamento de Newton e de StefanBoltzmann, tratam, respectivamente, de processos de condução, convecção e radiação; que constituem as três formas pela qual o calor é transferido entre dois corpos que se encontrem em temperaturas distintas. Cabe aqui ressaltar que, em sistemas físicos reais, os três mecanismos de transferência de calor estão presentes; e, consequentemente, o calor transferido é resultado da soma das contribuições correspondentes a cada um deles [7].

\subsection{Transferência de calor por convecção}

Na convecção, o produto do coeficiente de película $h$ pela área da superfície sólida $A$ e pela diferença entre a temperatura da superfície sólida $T_{s}$ e do fluido circundante $T_{\infty}$ corresponde à taxa de transferência de calor $q_{c}$. Essa definição descreve a lei resfriamento de Newton, cuja expressão analítica é dada pela equação (1):

$$
q_{c}=h A\left(\mathrm{~T}_{\mathrm{s}}-\mathrm{T}_{\infty}\right)
$$

A estimativa do valor do coeficiente de película constitui o problema da convecção, pois $h$ é um parâmetro empírico, determinado através de correlações que envolvem, entre outros, os números adimensionais de Nusselt $\left(N_{n u}\right)$, Reynolds $\left(N_{r e}\right)$ e Prandtl $\left(N_{p r}\right)$. Muitas dessas correlações são obtidas na literatura, devendo-se destacar que seu emprego é limitado a situações específicas e o grau de precisão dos resultados obtidos poderá variar em função da geometria do sólido e das características do escoamento [8]. Cabe acrescentar que, quando se avalia a transferência de calor entre uma superfície sólida e um fluido, o processo de convecção envolve os efeitos combinados de condução e movimento do fluido que ocorrem na interface que delimita esses dois meios materiais [9].

\subsection{Convecção livre (ou natural) do calor}

A convecção natural é um processo induzido pelas forças de empuxo que se originam das diferenças de densidade do fluido em pontos distintos ao longo da interface sólida. Correlações baseadas nos números de Grashoff $\left(N_{g r}\right)$, que corresponde ao quociente entre o empuxo e as forças viscosas, são utilizadas para estimar o valor do coeficiente de película. O valor dessa grandeza adimensional é dado pela equação (2):

$$
N_{g r}=\frac{g \beta\left(T_{s}-T_{\infty}\right) L_{c}^{3}}{\gamma^{2}}
$$

na qual $g, L_{c}, \beta$ e $\gamma$ correspondem, respectivamente, à aceleração da gravidade, ao comprimento característico da interface sólida objeto de análise, ao coeficiente de expansão térmica, e à viscosidade cinemática do ar na temperatura do filme. Correlações envolvendo os números de Nusselt $\left(N_{n u}\right)$, Prandtl $\left(N_{p r}\right)$ e Rayleigh $\left(N_{r a}\right)$ na convecção natural sobre uma placa plana vertical são dadas pelas equações (3) a (6) [10]:

$$
\begin{gathered}
N_{n u}=\left\{0,825+\frac{0,387 N_{r a}^{1 / 6}}{\left[1+\left(\frac{0,492}{N_{p r}}\right)^{9 / 16}\right]^{8 / 27}}\right\}^{2} \\
N_{n u}=\frac{h L_{c}}{k_{f}} \\
N_{p r}=\frac{\gamma}{\alpha} \\
N_{r a}=N_{g r} N_{p r}
\end{gathered}
$$

nas quais $\alpha$ é a difusividade térmica do sólido; $h$, o coeficiente de película na interface sólida, e $k_{f}$ é a condutividade térmica do ar na temperatura de filme. Essa temperatura varia ao longo do processo de resfriamento e corresponde à média entre o valor da temperatura do fluido $\left(\mathrm{T}_{\infty}\right)$ e a temperatura instantânea do sólido $\left(\mathrm{T}_{\mathrm{S}}\right)$, sendo calculada conforme a equação (7):

$$
T_{\text {filme }}=\frac{T_{s}+T_{\infty}}{2}
$$

Uma placa plana horizontal pode constituir a interface superior ou inferior de um objeto sólido, delimitando, respectivamente, a base ou o topo desse objeto e possuir uma temperatura superior ou inferior à do fluido circundante. Quando essa interface for a superfície superior de uma placa quente, utiliza-se as correlações dadas pelas equações (8) e (9):

$$
\begin{aligned}
& N_{n u}=0,54 N_{r a}^{1 / 4} \text { para } 10^{4}<N_{\mathrm{ra}}<10^{7} \\
& N_{n u}=0,15 N_{r a}^{1 / 3} \text { para } 10^{7}<N_{\mathrm{ra}}<10^{11}
\end{aligned}
$$

Quando essa interface for a superfície inferior de uma placa quente, utiliza-se a correlação dada pela equação (10):

$$
N_{n u}=0,27 N_{r a}^{1 / 4} \text { para } 10^{5}<N_{\text {ra }}<10^{11}
$$

No cálculo do coeficiente de película para uma placa plana horizontal, de formato circular, o comprimento característico da interface sólida corresponde à razão entre a área e o perímetro da placa.

\subsection{Transferência de calor por radiação}

Todas as formas de matéria emitem energia, cujo transporte ocorre na forma de ondas eletromagnéticas e sem que haja necessidade de um material para sua propagação [3]. A quantidade de energia irradiada é função da temperatura do material, sendo que tal radiação é constituída 
por uma distribuição contínua e não-uniforme do comprimento de onda $(\lambda)$. A radiação, consequentemente, possui dependência espectral; e, também, direcional, pois sua intensidade varia com a direção na qual é emitida $[11,12,13]$.

O corpo que, a uma dada temperatura, emite a máxima radiação possível é conceituado como corpo negro; sendo que a razão entre a emissão de uma superfície real e a de um corpo negro (superfície ideal), numa dada temperatura, é denominada emissividade $(\varepsilon)$. O parâmetro $\varepsilon$, além de possuir dependência espectral e direcional, características da irradiação, irá variar com a temperatura e as características do material e o formato e a textura de sua superfície.

A lei de Stefan-Boltzmann nos diz que a taxa de transferência de calor por radiação entre uma superfície sólida e o fluido circundante é dada pela equação (11):

$$
q_{r}=\varepsilon \sigma A\left(T_{\infty}^{4}-T_{s}^{4}\right)
$$

na $\sigma$ qual é a constante de Stefan-Boltzmann e A é a área superficial do sólido.

\section{Descrição do experimento}

O experimento consiste no monitoramento da temperatura de um cilindro de aço, que é aquecido em uma mufla até a temperatura de $200{ }^{\circ} \mathrm{C}$, e posteriormente exposto ao ambiente. Durante a realização do ensaio, esse objeto permanece suspenso verticalmente por um barbante em uma estrutura metálica. No cilindro existe um furo central e outro posicionado junto à sua borda, nos quais são introduzidos dois sensores de temperatura, do tipo PT 100, que são conectados a um módulo de aquisição de dados.

O módulo de aquisição de dados utilizado possui oito canais analógicos, que permite o registro simultâneo de oito sensores, que transformam grandezas físicas, como temperatura, pressão, força, deslocamento e outras, em sinais elétricos. Esse sinal é convertido em um registro digital e armazenado na forma de arquivos eletrônicos. Assim, foi utilizado um sistema de aquisição de dados, integrado por um módulo com oito canais, sensores do tipo PT 100 e um notebook, no qual foi instalado um softwaredisponibilizado com a compra desse módulo - que permite a visualização da temperatura, em tempo real e em uma tela de computador, dos valores mensurados durante o ensaio. Além disso, é possível selecionar o intervalo entre registros - que pode ser até alguns milissegundos - e, ao final dos ensaios, transferir os dados armazenados para planilhas eletrônicas; facilitando a realização de operações matemáticas, filtragem, seleção e formatação dos dados, e confecção de relatórios. Na Figura 1 são apresentados o módulo de aquisição de dados e o sensor de temperatura.

Na Figura 2 são apresentadas a estrutura metálica utilizada para manter o cilindro de aço suspenso no ar e uma imagem desse cilindro, com os furos nele produzidos

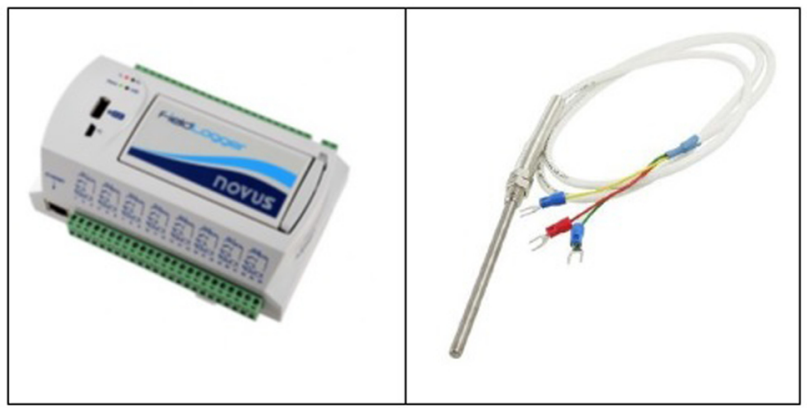

Figura 1: Imagem do módulo de aquisição de dados (esquerda) e de um sensor de temperatura do tipo PT100 (direita)

para inserção dos sensores de temperatura. Cabe aqui destacar que essa estrutura foi projetada para também ser utilizada em outros experimentos.

Nesse experimento são mensuradas as temperaturas do ar ambiente e do cilindro de aço. A comparação entre as temperaturas da borda e do centro do cilindro, que pode ser feita visualmente no decorrer do ensaio, permite verificar a hipótese de temperatura uniforme nesse sólido, conforme será detalhado na seção 4 .

$\mathrm{O}$ cilindro de aço utilizado nesse experimento foi pintado com tinta preta, cuja emissividade situa-se na faixa de 0,95 a 0,99 , de acordo com a literatura consultada $[14,15,16,17]$. Esse procedimento foi adotado porque a variação relativa da emissividade para uma superfície revestida com tinta preta é muito pequena; permitindo quantificar, com maior precisão, o calor irradiado pelo cilindro.

Após a realização do experimento, realiza-se a transferência dos dados armazenados no módulo de aquisição de dados, obtendo-se uma planilha eletrônica com registros sequenciais de temperatura. Essa planilha será utilizada para realização dos cálculos e na análise da consistência dos resultados experimentais frente ao modelo teórico utilizado para avaliar o comportamento do sistema.

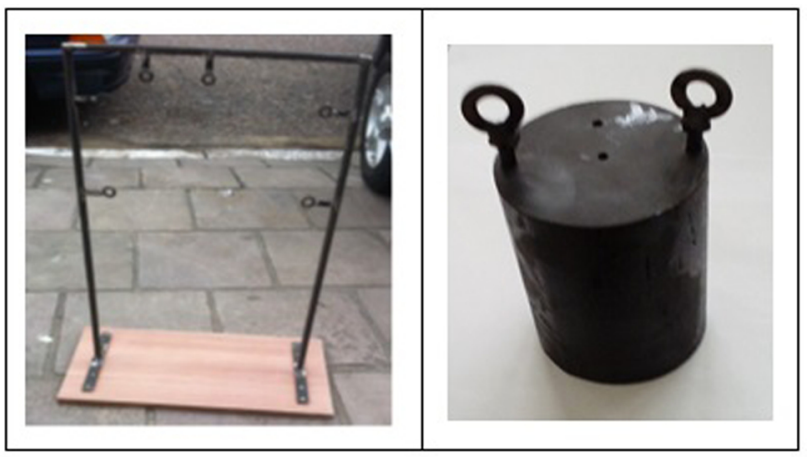

Figura 2: Estrutura metálica utilizada para suspensão do cilindro de aço (esquerda) e imagem ampliada desse cilindro (direita) 


\section{Metodologia utilizada}

Nesse experimento busca-se quantificar a transferência de calor durante o resfriamento de um cilindro de aço. Um balanço térmico desse objeto nos permite afirmar que o calor $(Q)$ cedido ao ambiente é proporcional à variação da temperatura $(\Delta T)$ do cilindro de aço, segundo a expressão 12 :

$$
Q=m C_{p} \Delta T
$$

na qual $m$ e $C_{p}$ são, respectivamente, a sua massa e o seu calor específico. A validade da equação 12 condiciona-se à hipótese de que a temperatura desse sólido é espacialmente uniforme. Essa hipótese é válida quando o número adimensional de Biot $\left(N_{b i}\right)$ for inferior a 0,1 [18]. Esse número adimensional consiste na razão entre a resistência térmica condutiva no seu interior do sólido e a resistência térmica convectiva na interface que o delimita, sendo calculado pela expressão 13 :

$$
N_{b i}=\frac{h L}{k}
$$

na qual $L$ é o comprimento característico do sólido - isto é, a razão entre o volume e a área superficial desse sólido - e $k$ é a sua condutividade térmica. No experimento foi utilizado um cilindro cujo raio e altura são, respectivamente, 36 e $102 \mathrm{~mm}$, confeccionado em aço carbono. O resfriamento desse cilindro ao ar consiste num processo de convecção natural, cujo coeficiente de película situase na faixa de 2 a $25 \mathrm{~W} /\left(\mathrm{m}^{2} . \mathrm{K}\right)$ [3]; e a condutividade térmica desse objeto é de $52 \mathrm{~W} /(\mathrm{m} . \mathrm{K})$, resultando num valor de Bi compreendido entre 0,000512 e 0,0064. Logo, para o experimento proposto, a equação 12 é válida.

O calor cedido pelo cilindro $(Q)$ é transferido, simultaneamente, por convecção e radiação, numa taxa que equivale à soma das taxas correspondentes a esses dois mecanismos de transferência. Logo, tal quantidade de calor pode ser igualada ao produto dessa soma pelo intervalo de tempo $(\Delta t)$ durante o qual se processa o resfriamento do sólido, conforme a equação (14):

$$
Q=\left(q_{\text {conv. }}+q_{\text {rad. }}\right) \Delta t
$$

na qual $q_{c o n v}$. e $q_{\text {rad. }}$ correspondem, respectivamente, às taxas de convecção e radiação do calor. Nesse ponto cabe ressaltar que o calor cedido pelo cilindro, Q1, é calculado com base nos valores de temperatura registrados durante o experimento, através da equação 15 :

$$
Q_{1}=m C_{p}\left(T_{i}-T_{f}\right)
$$

sendo $T_{i}$ e $T_{f}$, respectivamente, a temperatura inicial e final do cilindro em cada intervalo de tempo. O calor transferido para o ar, Q2, é calculado com base em equações teóricas de convecção e radiação do calor, através da equação 16 :

$$
\begin{array}{r}
Q_{2}=Q_{\text {rad. }}+Q_{\text {conv. }}=\left[h A\left(\mathrm{~T}_{\mathrm{s}}-\mathrm{T}_{\infty}\right)\right. \\
\left.+\varepsilon \sigma A\left(T_{s}^{4}-T_{\infty}^{4}\right)\right] \Delta t
\end{array}
$$

Neste ponto cabe atentar para o fato de que a temperatura da interface sólida não é constante e, consequentemente, as taxas de transferência de calor por radiação e convecção variam ao longo do processo. Dessa forma, a equação 16 só é válida para um intervalo de tempo suficientemente pequeno, durante o qual a variação dessas taxas seja desprezível.

\subsection{Elaboração da planilha eletrônica}

Utilizando as temperaturas registradas na planilha eletrônica obtida após a realização de um ensaio, aplica-se o balanço térmico expresso na equação (14) para um intervalo de tempo fixado. A Tabela 1 indica as dimensões e as propriedades termofísicas do cilindro de aço utilizado no experimento.

O ensaio a seguir descrito consistiu no resfriamento de um cilindro, inicialmente a $200{ }^{\circ} \mathrm{C}$, até a temperatura de $100{ }^{\circ} \mathrm{C}$. Esse intervalo de temperatura foi selecionado de modo que não houvesse uma diferença significativa entre as quantidades de calor transferidas por convecção e radiação; de modo a não ampliar os desvios registrados entre resultados teóricos e experimentais. No balanço térmico desse processo foi adotado um intervalo de tempo de 1 minuto. $O$ valor de $\varepsilon$ empregado foi de 0,97 , que corresponde ao valor médio desse parâmetro para uma superfície pintada de preto, conforme relatado na seção 3. Durante a realização do ensaio, a temperatura do ar era de $23^{\circ} \mathrm{C}$.

O coeficiente de película teórico foi calculado para cada uma das interfaces sólidas que delimitam o cilindro, a saber: a superfície lateral do cilindro - tratada como uma placa plana vertical - e as superfícies de topo e da base desse cilindro que constituem, respectivamente, uma superfície superior e inferior de placa horizontal aquecida. A rotina de cálculo do h teórico é apresentada a seguir.

Inicialmente, são calculadas as propriedades termofísicas do ar no início e no final do processo de resfriamento do cilindro, com base em valores tabelados [3]. Na Tabela 2 são apresentados os valores obtidos.

Numa segunda etapa são calculados os números adimensionais, o comprimento característico e o valor correspondente ao coeficiente de película teórico para cada uma das três interfaces sólidas, no início e no final do processo de resfriamento, com o emprego das equações apresentadas na seção 2.2 desse artigo. Na Tabela 3 são apresentados os valores obtidos.

Tabela 1: Características do cilindro de aço.

\begin{tabular}{lll}
\hline Altura do cilindro $(\mathrm{mm})$ & $\mathrm{H}$ & 102 \\
\hline Raio do cilindro $(\mathrm{mm})$ & $\mathrm{R}$ & 36 \\
\hline Massa específica $\left(\mathrm{kg} / \mathrm{m}^{3}\right)$ & $\mathrm{P}$ & 7860 \\
\hline Área superficial $\left(\mathrm{m}^{2}\right)$ & $\mathrm{A}$ & 0,0312 \\
\hline Massa $(\mathrm{kg})$ & $\mathrm{M}$ & 3,264 \\
\hline Comprimento característico do cilindro $(\mathrm{m})$ & $\mathrm{L}$ & 0,0133 \\
\hline Condutividade térmica $(\mathrm{W} /(\mathrm{m} . \mathrm{K}))$ & $\mathrm{K}$ & 52 \\
\hline Calor específico $(\mathrm{J} /(\mathrm{kg} . \mathrm{K}))-273 \mathrm{~K}$ & $\mathrm{C}_{\mathrm{p}}$ & 470 \\
\hline
\end{tabular}


Numa terceira e última etapa, são estimados os coeficientes de película teórico inicial e final do processo de resfriamento. Tais coeficientes são obtidos como uma média ponderada, em relação à área, dos valores de h teóricos nas três interfaces sólidas que delimitam o cilindro. A metodologia de cálculo do h médio se baseia no fato de que a taxa de transferência instantânea de calor é constituída pelo somatório das contribuições individuais de cada interface, sendo que essas contribuições são o produto da área pelo coeficiente de película da respectiva interface, e pela diferença de temperatura entre o cilindro e o ar. Na Tabela 4 são apresentados os valores resultantes desses cálculos, destacando que o h inicial corresponde ao valor máximo desse coeficiente, pois esse parâmetro decresce com a diminuição da temperatura de filme.

O balanço térmico expresso nas equações 150 e 116 é esquematizado na Figura 3, na qual $Q 1$ é a variação da energia térmica do cilindro. $\mathrm{O}$ calor cedido ao ambiente é constituído por duas parcelas, convecção e radiação, quantificadas nos dois termos presentes no membro direito de 16 e a partir dos coeficientes de película teóricos e da emissividade da superfície do cilindro. Essas duas parcelas, identificadas como $Q_{\text {rad. }}$ e $Q_{\text {conv }}$. na Fig. 3, são somadas e o resultado dessa soma, denotado por Q2, é confrontado com os valores calculados para $Q 1$, em cada intervalo de tempo do processo de resfriamento do cilindro.

$\mathrm{Na}$ Tabela 5 são apresentados os resultados dos intervalos iniciais do ensaio realizado. A temperatura do cilindro, expressa nessa tabela, corresponde à média entre a temperatura registrada na borda e no centro desse objeto. O cálculo de $Q 1$ foi feito com base nessa temperatura, que também foi utilizada para calcular o calor específico do cilindro em cada intervalo de tempo. No cálculo de Q2 é empregada a temperatura da borda do cilindro. Na coluna da direita da Tabela 5 também é apresentado o valor absoluto da diferença percentual, nos intervalos iniciais, entre os valores experimentais e teóricos do calor transferido na superfície do cilindro, isto é, entre $Q 1$ e $Q 2$, respectivamente. Observa-se que essas diferenças oscilam entre 11 e $13 \%$.

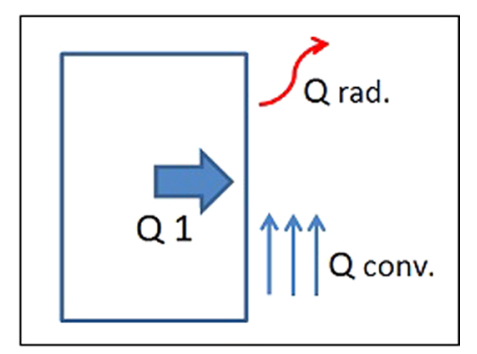

Figura 3: Esquema ilustrativo da metodologia adotada no cálculo do calor transferido durante o processo de resfriamento do cilindro. Os símbolos Q1, Qrad. e Qconv. correspondem, respectivamente, às quantidades de calor cedidas pelo cilindro (calculadas com base em dados experimentais) e as quantidades de calor transferidas por radiação e convecção, em cada intervalo de tempo (calculadas a partir de equações teóricas da radiação e convecção do calor)

Tabela 4: Valores teóricos do coeficiente de película do cilindro.

\begin{tabular}{lccc}
\hline $\begin{array}{l}\text { Superfície } \\
\text { do cilindro }\end{array}$ & Área $\left(\mathrm{m}^{2}\right)$ & $\mathrm{h}$ mínimo & $\mathrm{h}$ máximo \\
\cline { 3 - 4 } & & $\left(\mathrm{W} / \mathrm{m}^{2} . \mathrm{K}\right)$ & $\left(\mathrm{W} / \mathrm{m}^{2} . \mathrm{K}\right)$ \\
\hline Lateral & 0,0231 & 6,97 & 8,08 \\
\hline Inferior & 0,0041 & 5,38 & 6,20 \\
\hline Superior & 0,0041 & 10,75 & 12,40 \\
\hline Total & 0,0312 & 7,25 & 8,40 \\
\hline
\end{tabular}

Tabela 5: Comparação entre valores teóricos e experimentais das quantidades de calor transferidas nos intervalos iniciais do processo de resfriamento do cilindro.

\begin{tabular}{ccccccc}
\hline & $\begin{array}{c}\mathrm{T}_{\text {cilindro }} \\
\left({ }^{\circ} \mathrm{C}\right)\end{array}$ & $\begin{array}{c}\mathrm{Q} 1 \\
(\mathrm{~J})\end{array}$ & $\begin{array}{c}\mathrm{Q}_{\text {conv. }} \\
(\mathrm{J})\end{array}$ & $\begin{array}{c}\mathrm{Q}_{\text {rad. }} \\
(\mathrm{J})\end{array}$ & $\begin{array}{c}\mathrm{Q} 2 \\
(\mathrm{~J})\end{array}$ & $\begin{array}{c}\text { Diferença } \\
(\%)\end{array}$ \\
\hline 0 & 186,66 & & & & & \\
\hline 1 & 183,29 & 5914 & 2941 & 3728 & 6669 & 11,33 \\
\hline 2 & 180,02 & 5728 & 2873 & 3598 & 6471 & 11,50 \\
\hline 3 & 176,89 & 5440 & 2812 & 3477 & 6290 & 13,51 \\
\hline 4 & 173,80 & 5373 & 2752 & 3362 & 6114 & 12,11 \\
\hline
\end{tabular}

No gráfico da Figura 4 são plotados os valores teóricos para o calor transferido por radiação e convecção em cada intervalo de tempo ( $\mathrm{Q}$ rad. $\mathrm{e} \mathrm{Q}$ conv., respectivamente) a soma dessas quantidades (Q2) e os valores correspondentes ao calor cedido pelo cilindro com base nos registros

Tabela 2: Propriedades termofísicas do ar na temperatura de filme.

\begin{tabular}{lccccccc}
\hline & $\mathrm{T} \infty\left({ }^{\circ} \mathrm{C}\right)$ & Ts $\left({ }^{\circ} \mathrm{C}\right)$ & Tfilme $\left({ }^{\circ} \mathrm{C}\right)$ & $\begin{array}{c}\beta \\
\left(\bullet 10^{3} \mathrm{~K}^{-1}\right)\end{array}$ & $\begin{array}{c}\nu \\
\left(\bullet 10^{6} \mathrm{~m}^{2} / \mathrm{s}\right)\end{array}$ & $\begin{array}{c}\alpha \\
\left(\bullet 10^{6} \mathrm{~m}^{2} / \mathrm{s}\right)\end{array}$ & $\begin{array}{c}\mathrm{kar}_{\text {ar }} \\
\left(\bullet 10^{3} \mathrm{~W} / \mathrm{m} . \mathrm{K}\right)\end{array}$ \\
\hline Inicial & 183 & 23 & 103 & 2,66 & 24,36 & 34,75 & 31,73 \\
\hline Final & 103 & 23 & 63 & 2,98 & 20,00 & 28,51 & 28,82 \\
\hline
\end{tabular}

Tabela 3: Valores de h, inicial e final, nas três interfaces sólidas do cilindro.

\begin{tabular}{llccccc}
\hline Interface & & $\mathrm{Pr}$ & $\mathrm{L}_{\mathrm{c}}(\mathrm{m})$ & $\mathrm{Gr}$ & $\mathrm{Nu}$ & $\mathrm{h}\left(\mathrm{W} / \mathrm{m}^{2} \cdot \mathrm{K}\right)$ \\
\hline Lateral & Inicial & 0,701 & 0,102 & $7,47.10^{6}$ & 25,97 & 8,08 \\
& Final & 0.702 & 0,102 & $6,2.10^{6}$ & 24,66 & 6,98 \\
\hline Inferior & Inicial & 0,701 & 0,018 & $4,1.10^{4}$ & 3,52 & 6,20 \\
& Final & 0,702 & 0,018 & $3,41.10^{4}$ & 3,36 & 5,38 \\
\hline \multirow{2}{*}{ Superior } & Inicial & 0,701 & 0,018 & $4,1.10^{4}$ & 7,03 & 12,40 \\
& Final & 0,702 & 0,018 & $3,41.10^{4}$ & 6,71 & 10,75 \\
\hline
\end{tabular}


de temperatura $(Q 1)$, durante o processo de resfriamento desse sólido entre 183 e $103{ }^{\circ} \mathrm{C}$. O cilindro leva 37 minutos para se resfriar, sendo registrados, no gráfico, valores correspondentes ao calor transferido a cada minuto. O gráfico assim obtido mostra que os valores de $Q 1$ e $Q 2$ permanecem relativamente próximos, sendo que o valor médio da diferença absoluta entre essas quantidades, ao longo de todo o processo, é de 14,65\%. Cabe aqui ressaltar que o calor específico do material utilizado (aço carbono) possui uma variação da ordem $25 \%$ no intervalo de temperatura em que se processa o resfriamento da peça; e, visando minimizar o erro produzido por essa oscilação foi realizado um ajuste linear desse parâmetro em função da temperatura.

Uma outra forma de avaliar os resultados experimentais é calcular um coeficiente de película baseado no calor convectivo, descontando-se do valor de $Q 1$ a parcela correspondente ao calor transferido por radiação, conforme explicitado na equação (17) a seguir:

$$
\begin{array}{r}
Q_{3}=\left[h_{\exp } A\left(\mathrm{~T}_{\mathrm{s}}-\mathrm{T}_{\infty}\right)\right] \Delta t=m C_{p}\left(T_{i}-T_{f}\right) \\
-\left[\varepsilon \sigma A\left(T_{s}^{4}-T_{\infty}^{4}\right)\right] \Delta t
\end{array}
$$

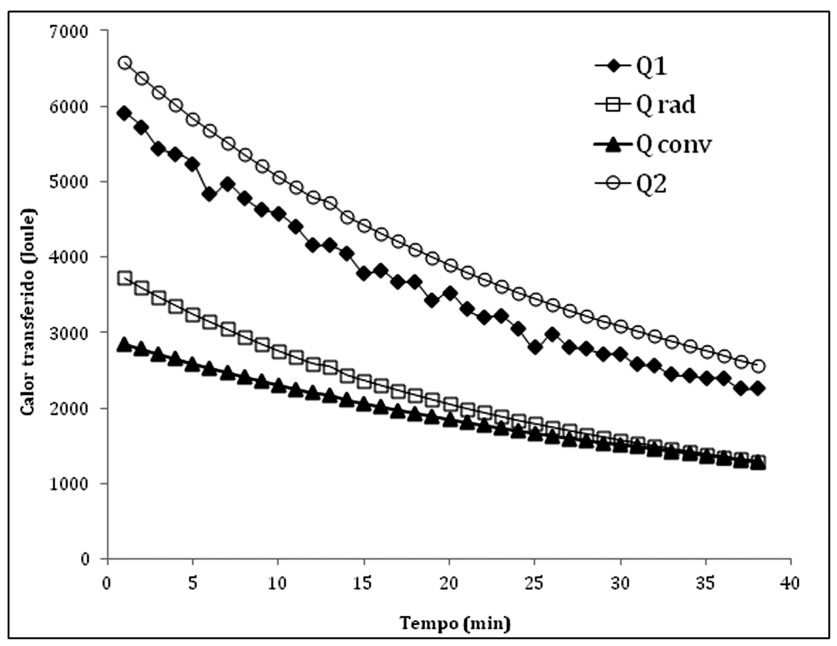

Figura 4: Gráfico comparativo entre as quantidades teóricas e experimentais de calor transferidas no processo, discriminando as parcelas correspondentes à convecção e radiação do calor. Q1 e Q2 correspondem, respectivamente ao calor cedido pelo cilindro em virtude de seu decréscimo de temperatura e ao calor teórico transferido para o ar. Q2 é dado pela soma das parcelas de calor transferidas por radiação e convecção ( $Q$ rad. e $Q$ conv., respectivamente)
As quantidades assim obtidas, denotadas como Q3, correspondem às parcelas convectivas de calor em cada intervalo de tempo e são associadas a um coeficiente de película experimental, $h_{\text {exp }}$.

Adotando-se procedimento similar ao empregado na elaboração do gráfico plotado na Figura 4, e utilizando os dados disponíveis nas Tabelas 2 a 5, elaborou-se a Tabela 6, na qual são comparados os valores teóricos e experimentais do coeficiente de película no início do processo de resfriamento. Cabe aqui recordar que o valor teórico desse coeficiente, em cada intervalo, é a média ponderada, em relação à área, do valor desse coeficiente nas três superfícies do cilindro.

Efetuando-se os cálculos para todo o período de realização do ensaio; isto é, até o resfriamento do cilindro na temperatura de $103{ }^{\circ} \mathrm{C}$, verifica-se que a média do valor absoluto das diferenças entre os dados teóricos e experimentais do coeficiente convectivo de transferência de calor, $h$, é igual a 15,63\%. A evolução desses valores ao longo do processo de resfriamento do cilindro é ilustrada no gráfico da Figura 5.

\section{Análise dos resultados}

Os resultados obtidos demonstraram que, nas condições em que o experimento foi realizado, os mecanismos de convecção e radiação são relevantes e nenhum deles pode ser descartado, conforme ilustrado na Figura 4. Nesse

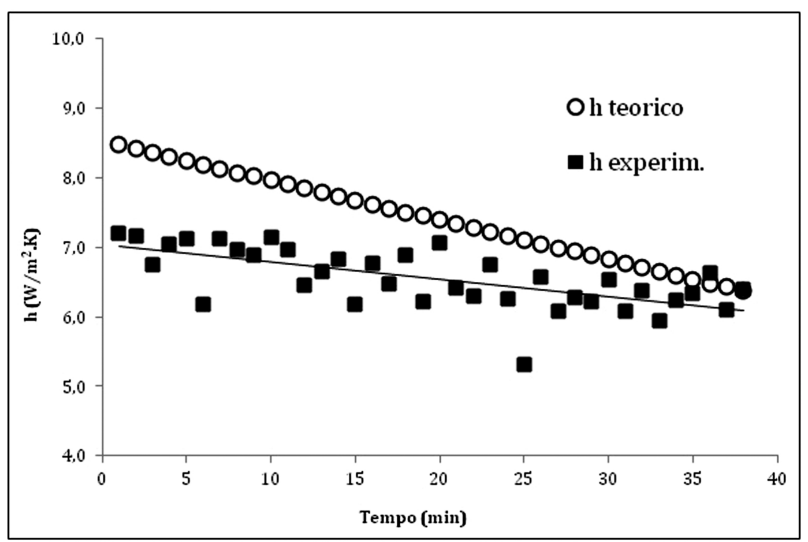

Figura 5: Gráfico comparativo entre o valor teórico e experimental do coeficiente de transferência convectivo de calor na interface sólida, ao longo do processo de resfriamento

Tabela 6: Comparação entre valores teóricos e experimentais do coeficiente de transferência convectiva de calor na interface na fase

\begin{tabular}{|c|c|c|c|c|c|c|c|}
\hline & $\begin{array}{c}\mathrm{T}_{\text {cilindro }} \\
\left({ }^{\circ} \mathrm{C}\right)\end{array}$ & $\begin{array}{l}\text { Q1 } \\
(J)\end{array}$ & $\begin{array}{c}\mathrm{Q}_{\text {radiação }} \\
(\mathrm{J})\end{array}$ & $\begin{array}{l}\text { Q3 } \\
(J)\end{array}$ & $\begin{array}{c}\mathrm{h}_{\text {experimental }} \\
\left(\mathrm{W} /\left(\mathrm{m}^{2} \cdot \mathrm{K}\right)\right)\end{array}$ & $\begin{array}{c}\mathrm{h}_{\text {teórico }} \\
\left(\mathrm{W} /\left(\mathrm{m}^{2} \cdot \mathrm{K}\right)\right)\end{array}$ & $\begin{array}{c}\text { Diferença } \\
(\%)\end{array}$ \\
\hline 0 & 186,66 & & & & & & \\
\hline 1 & 183,29 & 5914 & 3728 & 2186 & 7,21 & 8,40 & 14,49 \\
\hline 2 & 180,02 & 5728 & 3598 & 2130 & 7,17 & 8,38 & 14,51 \\
\hline 3 & 176,89 & 5440 & 3477 & 1963 & 6,74 & 8,36 & 19,31 \\
\hline 4 & 173,80 & 5373 & 3362 & 2012 & 7,05 & 8,32 & 15,28 \\
\hline
\end{tabular}
inicial do processo. 
ponto cabe destacar que alguns experimentos que buscam avaliar a transferência de calor em processos transientes baseados em modelos globais - isto é, nos quais a temperatura da superfície do sólido varia com o tempo, mas os gradientes internos de temperatura são desprezíveis - consideram apenas o mecanismo convectivo de transferência de calor $[8,19,20,21]$. Essa simplificação é válida apenas quando a emissividade da superfície sólida e a diferença de temperatura na interface entre o sólido e o fluido são baixas.

No experimento ora descrito, a utilização de um sistema de aquisição de dados e o emprego de sensores de temperatura inseridos em furos presentes no cilindro de aço possibilitou a realização de medições em intervalos de tempo da ordem de frações de segundo. Além disso, na análise relativa ao fenômeno da convecção do calor na superfície sólida, o cilindro de aço foi avaliado como sendo constituído de três superfícies distintas, de forma a estimar corretamente o calor convectivo cedido por cada uma delas. Tais procedimentos tendem a minimizar as discrepâncias entre valores teóricos e experimentais correspondentes ao coeficiente de película $(h)$ e o calor global transferido entre o cilindro e o ar. Contudo, vários fatores tendem a induzir erros experimentais ou alterações no processo de resfriamento de cilindro, obtendo-se alguma discordância entre os valores previstos pelas equações que descrevem o comportamento térmico desse sistema e os valores calculados com base em dados experimentais. Os autores apontam alguns fatores responsáveis por essas discrepâncias:

a. Problemas na medição e registro da temperatura do corpo sólido e do ar ambiente;

b. Perturbações no escoamento do ar junto à superfície do cilindro;

c. Emprego de correlações inadequadas para estimativa de um valor teórico do coeficiente de película;

d. Incerteza nos valores utilizados para as propriedades termofísicas do cilindro;

e. Dificuldades na quantificação do calor transferido por radiação.

f. O aumento da área de transferência de calor, em virtude da inserção de anéis metálicos para facilitar a suspensão do cilindro aquecido;

g. A transferência de calor pelas hastes dos sensores de temperatura inseridos no cilindro.

As dificuldades na quantificação do calor irradiado por um sólido residem, fundamentalmente, no grau de incerteza associado à sua emissividade. No experimento foi utilizado um cilindro pintado de preto, conforme relatado na seção 3 , de modo a minimizar o erro associado a essa quantificação.

O processo de convecção natural induz o escoamento de ar junto à superfície do sólido, cabendo ressaltar que esse escoamento pode sofrer a interferência de correntes de ar próximas ao objeto. Nos cálculos do coeficiente de película são empregadas três equações, que correspondem às três superfícies que delimitam o cilindro, não sendo consideradas as interferências que ocorrem entre o escoamento de ar induzido em cada uma dessas superfícies. Além disso, ocorrem interferências produzidas pela proximidade ou movimentação de pessoas e objetos. Tais interferências tendem a reduzir a movimentação do ar nessas superfícies e, consequentemente, o coeficiente de película obtido experimentalmente será menor que o valor teórico correspondente, conforme se verifica no gráfico apresentado na Figura 5. A quantidade de calor transferida por radiação também pode ser afetada pela presença de pessoas ou objetos em posição próxima ao cilindro. Contudo, no experimento realizado não foram dispensados maiores cuidados para controlar as perturbações produzidas no escoamento do ar ou no calor irradiado pelo cilindro, tendo em vista que o experimento foi concebido para constituir uma aula prática, na qual a execução dos ensaios seja realizada por um grupo de alunos.

\section{Conclusões}

A modelagem do processo de resfriamento do cilindro a partir de um balanço térmico, no qual foi considerada a transferência simultânea de calor por convecção e radiação, é um dos aspectos mais relevantes desse trabalho, que descreve um experimento destinado a capacitar o aluno a avaliar quantitativamente a transferência de calor em um sistema físico real. Dessa forma, não foi aplicado um modelo de capacitância global que avalia o resfriamento do objeto sólido baseado numa equação exponencial de decaimento da temperatura, empregada para calcular um coeficiente de película associado ao escoamento do fluido na interface sólida. Quando se adota esse tipo de equação para quantificar o processo de resfriamento de um sólido imerso em um fluido, é obtido um coeficiente de película aparente, no qual passam a ser incorporados os mecanismos de convecção e radiação.

No experimento descrito nesse trabalho, as quantidades de calor transferidas por convecção e radiação são calculadas de forma independente, obtendo-se uma diferença absoluta média de $15,63 \%$ entre o valor teórico e o experimental do coeficiente de película.

A utilização de um sistema de aquisição de dados possibilitou uma investigação mais detalhada do processo de resfriamento do sólido, propiciando aos alunos uma melhor compreensão dos fenômenos associados à transferência de calor nesse sistema. O emprego de uma planilha eletrônica, na qual já estão inseridos os registros de temperatura obtidos na execução, constitui uma facilidade adicional que simplifica a realização dos cálculos e a elaboração de gráficos e tabelas. Dessa forma, é possível avaliar ensaios subdivididos numa extensa série de intervalos de tempo, e reduzir o tempo dispensado nos cálculos de parâmetros e grandezas físicas vinculadas ao ensaio realizado.

Diante do exposto, os autores concluem que o emprego de um sistema de aquisição de dados possibilita - a partir 
de um experimento simples e de fácil execução - uma investigação quantitativa adequada para o fenômeno físico estudado; e se constitui numa alternativa de baixo custo em relação ao emprego de um módulo didático comercial destinado ao estudo da convecção natural ao ar.

Cabe acrescentar que esse tipo de sistema pode ser utilizado em outros tipos de experimentos, que envolvem a quantificação de outras grandezas físicas além da temperatura, tais como pressão e umidade do ar. Nesse contexto, o trabalho aqui relatado pode servir de ponto de partida para a elaboração de ensaios similares, cujos esforços empregados na concepção e na formulação dos procedimentos podem ser compensados pelos conhecimentos adquiridos pelos alunos numa aula prática dessa natureza. A possibilidade de realizar uma investigação mais ampla dos fenômenos físicos envolvidos no ensaio; e, simultaneamente, a execução desse tipo de ensaio sem o emprego de equipamentos didáticos padronizados - cuja compra, em muitas situações, é inviabilizada por entraves burocráticos e financeiros - são aspectos relevantes do tipo de experimento proposto nesse trabalho.

Finalmente, cabe destacar que as diferenças verificadas entre os dados teóricos e experimentais apresentados nesse trabalho indicam que o modelo matemático adotado para simular o comportamento térmico do sistema é adequado. Além disso, os desvios constatados são compatíveis com as incertezas presentes na quantificação das propriedades termofísicas do sólido e nas estimativas de alguns parâmetros do sistema, tais como o coeficiente de película e a emissividade da superfície. Isso significa que a incerteza dos dados de entrada, utilizados no cálculo do calor transferido e do coeficiente de película teórico, situa-se na mesma faixa percentual dos desvios verificados entre valores teóricos e experimentais. Nesse sentido, os desvios entre os dados de entrada e saída são compatíveis e, consequentemente, o modelo adotado fornece resultados satisfatórios para o processo físico analisado.

\section{Agradecimentos}

Os autores gostariam de agradecer à Fundação de Amparo à Pesquisa do Estado do Rio Grande do Sul (FAPERGS) pelo suporte financeiro a esse projeto.

\section{Referências}

[1] P. Luismar, in: $X$ CONEEQ, Florianópolis, 2000, disponível em http://www.hottopos.com/regeq10/ luismar.htm

[2] L.D.F. Marczak, I.C. Tessaro, N.S.M. Cardozo e K. Wada, in: Anais do XXXI COBENGE, Rio de Janeiro, 2003, v. 1, p. 1-7.

[3] F.P. Incropera e D.P. de Witt, Fundamentos de Transferência de Calor e Massa (Ed. LTC, Rio de Janeiro, 2008).

[4] B.L. Fernandes, in: Anais do XLI COBENGE, Gramado, 2013, p. 1-6.
[5] J.V.H. D'Angelo e R.J. Zemp, Revista Ensino Superior Unicamp 13, 6 (2014).

[6] R.L. Garcia, D.A. Falcão, J.A. Della Giustina Neto, C. Pibernat e J.R. Zabadal, Revista Eletrônica Científica da UERGS 2, 54 (2016).

[7] A. Bejan, Transferência de Calor (Ed. Edgard Blucher, São Paulo, 1996), p. 570.

[8] A. Antonietti, A. Stempkowski, F. Segatti, T. Spassin e C. Silva, Perspectiva 130, 7 (2011).

[9] Y.A. Çengel e A.J. Ghajar, Transferência de Calor e Massa: Uma Abordagem Prática (Ed. McGraw-Hill, Porto Alegre, 2012).

[10] S.W. Churchill and H.H. Chu, Int. J. Heat Mass Tran. 18, 1049 (1975).

[11] J.P. Holman, Transferência de Calor (Ed. Mc Graw-Hill, São Paulo, 1983).

[12] R. Siege and J.R. Howell, Thermal Radiation Heat Transfer (Ed. Taylor \& Francis, Washington, 1992).

[13] G.G.D. Teixeira, Confiabilidade Metrológica em Termografia Aplicada em Sistemas Elétricos. Dissertação de Mestrado, Universidade Federal de Minas Gerais, 2012.

[14] M.A. Bramson, Infrared Radiation - A Handbook for Applications (Ed. Plenum Press, New York, 1968).

[15] J.F. Kreider and F. Kreith, Principles of Solar Energy Handbook (Ed. Hemisphere Publishing Co., New York, 1978).

[16] Y.S. Toloukian and C.Y. Yo, Thermophysical Properties of Matter (Ed. Plenum Press, New York, 1972).

[17] W.L. Wolfe and G.J. Zissis, The Infrared Handbook (Office of Naval Research, Washington, D.C., 1989).

[18] C.R. Maliska, Transferência de Calor e Mecânica dos Fluidos Computacional (Ed. LTC, Rio de Janeiro, 2004).

[19] G.E. Amiozzo e L. Menoncini, Condução Unidimensional do Calor em Regime Estacionário. Monografia, Universidade Comunitária da Região de Chapecó, 2011.

[20] E.J.P.M. Júnior e R.S. Gonçalves, Revista ETC Educação, Tecnologia e Cultura 14, 1 (2016).

[21] F.R. Naia, A.L.F.L. Silva e S.M.M.L. Silva, in: Anais do XIV CREEM, Uberlândia, 2007, p. 1-10. 